University of Konstanz

Department of Economics

\title{
Risk Taking with Additive and Multiplicative Background Risks
}

Günter Franke, Harris Schlesinger, and Richard C. Stapleton

Working Paper Series

2011-25 


\title{
Risk Taking with Additive and Multiplicative Background risks.
}

\author{
Guenter Franke ${ }^{1}$, Harris Schlesinger ${ }^{2}$, Richard C. Stapleton, ${ }^{3}$
}

September 25, 2010

\footnotetext{
${ }^{1}$ Univerity of Konstanz, Germany, guenter.franke@uni-konstanz.de

${ }^{2}$ University of Alabama, USA, hschlesi@cba.ua.edu

${ }^{3}$ University of Manchester UK., richard.stapleton1@btinternet.com Valuable comments from Carsten Sorensen as well as from seminar participants at the EGRIE meeting in Zurich, and at the Universities of Pensylvania, Melbourne, Manchester, and Lancaster are gratefully acknowledged
} 


\begin{abstract}
Risk Taking with Additive and Multiplicative Background risks.

We examine the effects of background risks on optimal portfolio choice. Examples of background risks include uncertain labor income, uncertainty about the terminal value of fixed assets such as housing and uncertainty about future tax liabilities. While some of these risks are additive and have been amply studied, others are multiplicative in nature and have received far less attention. The simultaneous effect of both additive and multiplicative risks has hitherto not received attention and can explain some paradoxical choice behavior. We rationalize such behavior and show how background risks might lead to seemingly U-shaped relative risk aversion for a representative investor.
\end{abstract}




\section{Introduction}

In this paper we ask how the combination of additive and multiplicative background risks affects behavior towards an independent market risk. Previous papers have analyzed the separate effects of an additive background risk and of a multiplicative background risk on the derived risk aversion of an agent and hence on risk taking. For example, Gollier and Pratt (1996) establish conditions for risk vulnerability: when an unfair additive background risk increases derived risk aversion. Similarly, Franke, Schlesinger and Stapleton (2006) establish conditions for multiplicative risk vulnerability: when a unit mean multiplicative background risk increases derived risk aversion.

An independent, additive, zero-mean background risk typically tends to make an agent more risk averse towards a market risk, leading to a more conservative policy. This is easily seen by using the notion of the derived utility, as introduced by Kihlstrom, Romer and Williams (1981) and Nachman (1981). Kimball (1993) and Gollier and Pratt (1996) derive conditions under which derived risk aversion increases when an additive background risk is introduced, which in turn leads to less risk taking in the market. Eeckhoudt, Gollier and Schlesinger (1996) derive conditions under which an increase in background risk raises derived risk aversion. Their conditions are related to those of Ross (1981), who considered the case of a background risk that had a zero correlation with market risk, although it might not be statistically independent.

The effect of a multiplicative background risk is less well researched. In general, this effect depends critically on the relative risk aversion function of the agent (Franke, Schlesinger and Stapleton (2006)). If utility is of the CRRA class, then it is well known that a non-hedgeable multiplicative risk will have no effect on risk taking. For example, Campbell and Viceira (2001) and Brennan and Xia (2002) analyse the effect of a multiplicative inflation risk on asset allocation. However, if the agent exhibits declining or increasing relative risk aversion, there may be significant effects of multiplicative risks

on asset allocation. If relative risk aversion is declining and convex, then a multiplicative background risk raises derived risk aversion. But if relative 
risk aversion is increasing and concave, it reduces derived risk aversion. ${ }^{1}$

Although the separate effects of additive, and to a lesser extent, multiplicative background risks are well established in the literature, the combined effects have not previously been derived. Here we assume the terminal wealth of the agent is composed of market wealth $x$ and non-market wealth $z$. In addition, we assume that the market wealth $x$ is subject to a multiplicative risk factor $y$. Hence, total wealth is given by $w=x y+z$.

Some examples illustrate this problem setup. (1) The investor may convert her terminal market wealth $x$, at the uncertain annuity rate $\tilde{y}$, into a lifetime annuity. Then $\tilde{z}$ represents income from non-market wealth, possibly including income from another job after retirement. This income is reduced by medical costs, insurance premia etc. and other regularly paid costs. (2) The investor living in a small country invests abroad and converts the terminal portfolio wealth, denominated in foreign currency, $x$ into home currency at the uncertain exchange rate $\tilde{y}$. Then $\tilde{z}$ represents non-portfolio wealth like housing, bequests etc., reduced by personal debt, in home currency. (3) $\tilde{y}$ may be a purchasing power index reflecting uncertain inflation. Then $\tilde{z}$ is non-market wealth in real terms, for example, the present value of a pension which is indexed to the purchasing power index.

In this paper, in line with much of the literature, we assume the agent has underlying preferences exhibiting constant relative risk aversion. Combining both, a risky non-market wealth and a multiplicative background risk, leads to a variety of results depending on the level of non-market wealth. For instance, suppose that non-market wealth has a negative mean. Then both an additive or a multiplicative background risk makes the CRRA agent act in a more risk averse manner. Also, the two risks together reinforce this effect. However, if non-market wealth has a positive mean, an additive background risk alone induces more risk-averse behavior of the investor; whereas a multiplicative background risk alone induces less risk-averse behavior. However, adding both risks simultaneously does not yield some convex combination of these two separate effects. Rather, it may yield an even stronger increase in risk aversion than the non-market wealth risk alone. Thus, the interac-

\footnotetext{
${ }^{1}$ See Franke, Schlesinger and Stapleton (2006). The intuition for this result is discussed in Section 3, below.
} 
tion of both background risks needs to be considered in modeling risk-taking decisions. The purpose of this paper is to explore these combined effects.

Given risky non-market wealth and the multiplicative background risk, it is the agent's derived risk aversion towards market risk that determines her optimal choice. ${ }^{2}$ We first analyse the combined effects of risky non-market wealth and multiplicative background risk on the derived relative risk aversion of the agent. We then derive the agent's optimal demand for statecontingent claims assuming a perfect market which is also complete regarding tradable claims.

The outline of this paper is as follows. In the following section, we set up the problem of maximising the expected utility of terminal wealth and determine the conditions for optimal choice given risky non-market wealth and a multiplicative background risk. In section 3 , we review the existing results on the effect of additive and multiplicative background risk on risk aversion. In section 4, we derive results showing the impact of a multiplicative risk, given the existence of risky non-market wealth. In Section 5, we illustrate our results by numerical examples of demand curves for state-contingent claims.

\section{Conditions for Optimal Risk Taking}

Consider a risk-averse agent who maximizes the expected utility of terminal wealth . For the sake of concreteness, we assume constant relative risk aversion, with marginal utility of wealth $w$ given by $u^{\prime}(w)=w^{-\gamma}$, where $\gamma>0$ denotes the degree of relative risk aversion. ${ }^{3}$

As discussed above, we assume that terminal wealth is given by

$$
\tilde{w}=\tilde{x} \tilde{y}+\tilde{z},
$$

where $\tilde{x}$ is the chosen market wealth, $\tilde{y}$ is a positive multiplicative risk factor and $\tilde{z}$ is the risky non-market wealth. Without loss of generality, $E(\tilde{y})=1$.

\footnotetext{
${ }^{2}$ See Nachman (1982) for the notion of derived risk aversion.

${ }^{3}$ As is well known in this case, utility takes the form $u(w)=\ln w$ for $\gamma=1$. Otherwise, $u(w)=(1-\gamma)^{-1} w^{1-\gamma}$.
} 
Market wealth $\tilde{x}$ depends on the market return, $\widetilde{R}, \tilde{x}=x(\widetilde{R})$. We further assume that $\widetilde{R}, \tilde{y}$ and $\tilde{z}$ are independent random variables. As shown in Appendix 1, this setup can be easily generalized for the case where the risks are correlated. ${ }^{4}$

Let

$$
\nu(x) \equiv E[u(x \tilde{y}+\tilde{z})]
$$

denote the derived utility of market wealth, $x .{ }^{5}$ Then the optimization problem can be written as

$$
\max _{x(R)} E[\nu(x(\widetilde{R}))], \text { s.t. } E[\phi(\widetilde{R}) x(\widetilde{R})]=x_{0},
$$

where $\phi(R)$ denotes the pricing kernel for the tradable claims, with $E[\phi(\tilde{R})]=$ $1 / R_{f}$ and where $R_{f}$ is the gross risk-free rate and $x_{0}$ is the value of the initial market endowment of claims.

The first-order conditions for optimizing (3) are the budget constraint together with the conditions

$$
\nu^{\prime}(x(R))=\lambda \phi(R), \forall R
$$

where $\lambda$ is the Lagrange multiplier. ${ }^{6}$ Differentiating (4) with respect to $R$ and then using (4) to replace $\lambda$ in the result, we obtain

$$
x^{\prime}(R)=\frac{\phi^{\prime}(R) / \phi(R)}{\nu^{\prime \prime}(x(R)) / \nu^{\prime}(x(R))} .
$$

Letting $A_{z, y}(x) \equiv-x \nu^{\prime \prime}(x) / \nu^{\prime}(x)$ denote the Arrow-Pratt measure of relative risk aversion for the derived utility, it follows from (5) that the optimal contingent claim $x(R)$ satisfies

$$
\left[A_{z, y}(x)\right]^{-1}=-\frac{x^{\prime}(R) \phi(R)}{x(R) \phi^{\prime}(R)}=-\frac{d \ln x(R) / d \ln R}{d \ln \phi(R) / d \ln R} .
$$

\footnotetext{
${ }^{4}$ Note that $R$ can be any risk factor on which contingent claims are traded.

${ }^{5}$ See Kihlstrom, Romer and Williams (1981), Nachman (1982).

${ }^{6}$ Note that for a CRRA agent her marginal utility covers the whole set of positive numbers. Therefore, given the usual integrability conditions, the optimal solution is interior (see Back and Dybvig (1993)).
} 
Hence

$$
\frac{d \ln x}{d \ln R}=\left[A_{z, y}(x)\right]^{-1} \cdot\left[-\frac{d \ln \phi}{d \ln R}\right] .
$$

Condition (7) always holds, i.e. regardless of the existence of non-market wealth and multiplicative risk. But the relative risk aversion $A_{z, y}(x)$ depends on those risks and so the optimal policy does also.

In the absence of non-market wealth $(z \equiv 0)$ and multiplicative risks $(y \equiv 1)$, $A_{z, y}(x)=\gamma$ due to our assumption of CRRA utility for total wealth. As our benchmark case, we also assume that the second term (i.e. the elasticity of the pricing kernel) is a positive constant. ${ }^{7}$ Then, $\ln x(R)$ is an increasing and linear function of $\ln R$. That is, the agent has a log-linear demand function for contingent claims. Moreover, this linear function is flatter, ceteris paribus, for a higher level of relative risk aversion. This case is our benchmark case when we analyze the agent's policy in the presence of non-market wealth and multiplicative risks.

\section{The Separate Effects of Additive and Mul- tiplicative Background Risks on Derived Risk Aversion: A Review}

In order to analyze the combined effects of additive and multiplicative background risks, we first need various results from the literature concerning their separate effects. These are used as building blocks in the subsequent analysis. These are all special cases of the general model in equation (1).

First, consider $A_{z}(x) \equiv A_{z, y}(x)$, given $y \equiv 1$, i.e. the derived relative risk aversion in the absence of multiplicative risk. Also, define $\bar{z} \equiv E(\tilde{z})$. We summarize the results in the literature as follows.

\footnotetext{
${ }^{7}$ This, in turn, follows if $R$ follows a Geometric Brownian Motion.
} 


\subsection{The Effect of Non-Stochastic Non-Market Wealth $[\tilde{z} \equiv \bar{z}, \tilde{y} \equiv 1]$}

Intuitively, if agents have positive, non-stochastic non-market wealth, their risk-taking behavior in the market will be more aggressive than in the absence of this wealth. In fact, we have the following results which are similar to those found in Bodie, Merton and Samuelson (1992):

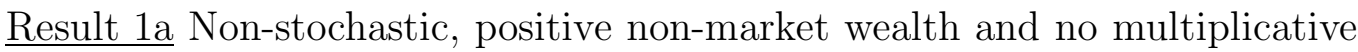
background risk [Bodie, et.al.]

Let $\tilde{z}=\bar{z}>0$ and $\tilde{y} \equiv 1$. Then an agent with CRRA preferences acts towards the market risk like an agent with HARA preferences 8 and derived relative risk aversion, $A_{z}(x)<\gamma$. Also, $A_{z}(x)$ is increasing in $x$.

$\underline{\text { Result } 1 \mathrm{~b}}$ Non-stochastic, negative non-market wealth and no multiplicative background risk [Bodie, et.al.]

Let $\tilde{z}=\bar{z}<0$ and $\tilde{y} \equiv 1$. Then an agent with CRRA preferences acts towards the market risk like an agent with HARA preferences and derived relative risk aversion, $A_{z}(x)>\gamma$. Also, $A_{z}(x)$ is decreasing in $x$.

\subsection{The Effect of Stochastic Non-Market Wealth, $\bar{z} \leq$ $0, \tilde{y} \equiv 1$}

There is an extensive literature (see Gollier (2001)) studying the effect of a non-positive-mean, independent background risk on derived risk aversion. This has direct implications for risk-taking behavior in the market. The following result follows from Kimball (1993):

$\underline{\text { Result } 2}$ Stochastic, non-positive-mean, independent $\tilde{z}, \tilde{y} \equiv 1$ [Kimball]

A CRRA agent with relative risk aversion $\gamma$, who is subject to an independent background risk, $\tilde{z}$, with $\bar{z} \leq 0$, acts towards the market risk like an agent with relative risk aversion $A_{z}(x)>\gamma$.

\footnotetext{
${ }^{8}$ For HARA (hyperbolic absolute risk aversion) -utility $v^{\prime}(x)=(x+b)^{-\gamma}$ for some constant $\mathrm{b}$ and relative risk aversion equal to $\gamma /(1+b / x)$.
} 
Now we summarize results on the effect of a multiplicative background in the absence of additive background risk.

\subsection{The Effect of Multiplicative Background Risk}

The effect of a unit-mean, independent multiplicative background risk has been studied in Franke, Schlesinger and Stapleton (2006). Although, for a CRRA agent, a multiplicative risk, $\tilde{y}$, has no effect on risk taking, we have the following relevant results:

$\underline{\text { Result } 3}$ Multiplicative background risk [Franke, et.al.]

a) Suppose an agent with declining, convex relative risk aversion and relative risk aversion greater than 1 is subject to a unit-mean multiplicative background risk, then the agent becomes more risk averse towards an independent market risk.

b) Suppose an agent with increasing, concave relative risk aversion and relative risk aversion greater than 1 is subject to a unit-mean multiplicative background risk, then the agent becomes less risk averse towards an independent market risk.

In this paper we assume throughout an agent with CRRA preferences. However, the above results are relevant since, given non-market wealth, the agent may act like someone with declining or increasing relative risk aversion and hence react to a multiplicative background risk by acting in a more (or less) risk averse manner, as we show below. The result in Franke, Schlesinger and Stapleton (2006) [FSS] is of central importance in this paper. However, the intuition behind the result is not obvious. While in the case of additive background risk a wide class of utility functions exhibit risk vulnerability, this is not true in the case of multiplicative background risk. For many util-

ity functions a multiplicative background risk induces more risk taking. To add some intuition as to why this is the case we now compare the cases of additive and multiplicative risks.

From Gollier and Pratt (1996), declining, convex absolute risk aversion is a sufficient condition for risk vulnerability. If $a(x)$ is absolute risk aversion and 
$\hat{a}(x)$ is derived absolute risk aversion given a zero mean additive background risk $\tilde{\varepsilon}$, then

$$
\hat{a}(x)-a(x)=E[a(x+\varepsilon)-a(x)]+\operatorname{cov}\left(\frac{u^{\prime}(x+\varepsilon)}{E u^{\prime}(x+\varepsilon)}, a(x+\varepsilon)\right) .
$$

If $a(x)$ is convex, the first term is positive. Also, if both $a(x)$ and $u^{\prime}(x)$ are declining, the covariance term is also positive. Hence $u$ is risk vulnerable. By an analogous argument, if relative risk aversion is declining and convex, then $u$ is multiplicative risk vulnerable. ${ }^{9}$ Now suppose absolute risk aversion is increasing and concave. Then equation (8) implies $\hat{a}(x)<a(x)$. Similarly, increasing and concave relative risk aversion implies that a multiplicative background risk lowers derived relative risk aversion. However, whereas increasing and concave absolute risk aversion is rather unrealistic, increasing and concave relative risk aversion is not. As pointed out above, if non-market income is positive and non-stochastic, the CRRA agent will act like an agent with increasing, concave relative risk aversion.

\section{The Combined Effects of Additive and Mul- tiplicative Background Risk}

We begin our analysis of the joint effects of multiplicative background risk and non-market wealth by considering the case where the expected value of non-market wealth is negative. For example, the agent has some debt whose precise value will be known and repaid at the terminal date. Although this may not be the most common or relevant case, it is the one where the analysis is straightforward and the results are easily understood. This is why we address this case first and the more relevant cases later.

\footnotetext{
${ }^{9}$ As FSS point out, we also require relative risk aversion greater than or equal to 1 in order to preserve marginal utility declining in $y$.
} 


\subsection{Case 1: Negative Expected Non-Market Wealth}

Result $1 \mathrm{~b}$ above shows that a CRRA agent, with relative risk aversion $\gamma$, and negative non-stochastic non-market wealth acts like a HARA agent with relative risk aversion greater than $\gamma$, which is declining in $x$. If non-market wealth is stochastic and has negative mean, we would expect the derived relative risk aversion, $A_{z}(x)$ again to exceed $\gamma$ and to be declining. This is confirmed in the following, where $z_{\text {min }}$ denotes the lowest outcome of $\tilde{z}^{10}$

Lemma 1 If $E(\tilde{z}) \equiv \bar{z} \leq 0$ and $\sigma_{z}>0$, then the derived relative risk aversion $A_{z}(x) \rightarrow \infty$ as $x \rightarrow-z_{\min }$ and $A_{z}(x)$ is declining, convex and approaches $\gamma$ as $x \rightarrow \infty$.

Proof: See Appendix 2.

Lemma 1 together with Result 3a has the following direct implication for the portfolio demand of a CRRA agent. Let $\frac{\partial \ln x}{\partial \ln R},\left[\frac{\partial \ln x}{\partial \ln R} \mid \tilde{z}\right],\left[\frac{\partial \ln x}{\partial \ln R} \mid \tilde{z}, \tilde{y}\right]$ denote the slope in the absence of non- market wealth and multiplicative background risk, [in the presence of non- market wealth, given no multiplicative background risk], [in the presence of non-market wealth and multiplicative background risk].

Proposition 1 A random non-market wealth with non-positive expected value induces the agent to choose a more conservative portfolio, as compared to the case without non-market wealth, and if the agent's level of CRRA: $\gamma \geq 1$, then a multiplicative risk induces the agent to choose an even more conservative portfolio, i.e.

$$
\frac{d \ln x}{d \ln R}\left|\tilde{z}, \tilde{y}<\frac{d \ln x}{d \ln R}\right| \tilde{z}<\frac{d \ln x}{d \ln R}, \quad \forall x
$$

Proof: Proposition 1 follows from Lemma 1 and Result 3a.

\footnotetext{
${ }^{10}$ More formally, $z_{\text {min }}$ denotes the highest level of $z$ such that $\operatorname{Prob}\left(\tilde{z} \geq z_{\min }\right)=1$.
} 
In Proposition 1, we define a 'more conservative' portfolio as one where the demand curve for contingent claims, $\frac{d \ln x}{d \ln R}$, has a flatter slope everywhere, i.e. the portfolio payoff is less sensitive to the market return. The right-hand inequality states that (non- positive) non- market wealth induces the agent to choose a more conservative portfolio. This follows from Lemma 1 . The lefthand inequality states that the agent becomes even more conservative when subject, in addition, to a multiplicative background risk. This latter result stems from the fact that for the derived utility function given non-market wealth, $A_{z}(x) \geq \gamma \geq 1$ and $A_{z}(x)$ is declining and convex. The conclusion in the Proposition therefore follows directly from Result 3a above.

In this case, where $\bar{z}<0$, all three effects of $\bar{z}, \sigma_{z}$, and $\tilde{y}$ work in the same direction. $\bar{z}<0$ by itself induces a more conservative policy. This is reenforced by the risk of non-market wealth, $\sigma_{z}>0$. Also the existence of a multiplicative risk $\tilde{y}$ again reinforces this effect.

\subsection{Case 2: Positive Non-Market Wealth}

For most agents, a more likely scenario is that the mean of non-market wealth is positive. We first consider the case where non-market wealth is always positive, but risky. The non-market wealth could come from a number of possible sources: labor income, real estate, or bequests, for example. How should you invest in contingent claims if you expect a large bequest sometime in the future, but have little idea as to the size of the bequest? The effect of the non-market wealth clearly depends on its risk. If the risk is small, in comparison with the expected value, then the derived risk aversion, $A_{z}(x)<$ $\gamma$, and Result 1 above implies that $A_{z}(x)$ is increasing in $x$.

If the risk is large, but the minimum value of non-market wealth is positive, it is again likely that $A_{z}(x)<\gamma$ and increasing in $x$. However, this is not always the case. ${ }^{11}$ The following Lemma characterizes $A_{z}(x)$ for $z_{\min }>0$.

\footnotetext{
${ }^{11}$ To prove that $A_{z}(x)$ may have a local maximum and a local minimum, it suffices to consider an example. Let $\tilde{z}$ be distributed symetrically around the mean of 30 according to $(1,6.25 \% ; 20,25 \% ; 30,37.5 \% ; 40,25 \% ; 59,6.25 \%)$. In each pair the first number denotes the realisation of $z$ and the second number its probability. Then for $\gamma=3, A_{z}(x)$ attains a local maximum at $x=6$ and a local minimum at $x=25$.
} 
Lemma 2 Suppose non-market wealth is strictly positive, $z_{\min }>0$.

1. $A_{z}(x) \rightarrow 0$ as $x \rightarrow 0$ and $\exists\left(x^{o}, x^{o o}\right)$ with $x^{o o}>x^{o}$ such that, for $x \leq x^{o}$ and for $x \geq x^{o o}, A_{z}(x)$ is increasing and concave. $A_{z}(x)$ approaches $\gamma$ as $x \rightarrow \infty$. Also, $A_{z}(x)<\gamma$ for all $x$.

2. If $\sigma_{z}$ is sufficiently small, then $A_{z}(x)$ is increasing and concave for all $x$.

Proof: See Appendix 2.

Lemma 2 part 2. establishes that the derived relative risk aversion, $A_{z}(x)$, is increasing and concave when the risk of non-market wealth is small. This mirrors the previous result in Lemma 1 for the case where non-market wealth is negative. Lemma 2 part 1 shows that the story is not so straightforward when $\sigma_{z}$ is large. There are ranges of $x$ over which $A_{z}(x)$ is increasing and concave, however it is possible that this is not true over some intermediate range.

The implications of Lemma 2 for portfolio policy are now stated in the following Proposition. Let $y_{\min }$ and $y_{\max }$ denote the lowest and highest possible outcomes of $y$. ${ }^{12}$ Hence $x \leq x^{o} / y_{\max }$ implies $x y \leq x^{o}, \forall y$ and $x \geq x^{o o} / y_{\min }$ implies $x y \geq x^{o o}, \forall y$.

Proposition 2 Suppose non-market wealth is strictly positive, $z_{\min }>0$.

1. Non-market wealth induces the agent to behave in a less conservative manner

$\frac{d \ln x}{d \ln R} \mid \tilde{z}>\frac{d \ln x}{d \ln R}, \forall x$.

This effect is reinforced by the existence of a multiplicative risk for $x \leq x^{o} / y_{\max }$ and for $x \geq x^{o o} / y_{\min }$ provided that $A_{z}(x y) \geq 1, \forall x y$,

$$
\frac{d \ln x}{d \ln R}\left|\tilde{z}, \tilde{y}>\frac{d \ln x}{d \ln R}\right| \tilde{z}, \forall x \leq x^{o} / y_{\max }, x \geq x^{o o} / y_{\min }
$$

\footnotetext{
${ }^{12}$ More formally, $y_{\min }\left[y_{\max }\right]$ denotes the highest [lowest] level of $y$ such that $\operatorname{prob}(\tilde{y} \geq$ $\left.y_{\text {min }}\right)=1\left[\operatorname{prob}\left(\tilde{y} \leq y_{\max }\right)=1\right]$
} 
2. If $\sigma_{z}$ is sufficiently small, then $\frac{d \ln x}{d \ln R}\left|\tilde{z}, \tilde{y}>\frac{d \ln x}{d \ln R}\right| \tilde{z}$ holds for every $x$, given $A_{z}(x, y) \geq 1 \forall x y$.

Proof: The Proposition follows directly from Lemma 2 and Result 3b.

As expected, an agent with positive non-market wealth follows a less conservative portfolio policy, in the absence of multiplicative risks. This follows since positive non-market wealth acts as a substitute for bonds. When the agent is also subject to a multiplicative background risk, she tends to become even less conservative. Hence the portfolio choice effects of the two risks tend to reinforce each other.

\subsection{Case 3: Positive Expected Non-Market Wealth, where the Minimum Non-Market Wealth is Nega- tive}

Perhaps the most likely case for many agents is where the expected value of non-market wealth is positive, but there is some chance that it might turn out to be negative. For example, the size of medical or education related liabilities may outweigh the positive benefits from bequests or property, in some future scenarios. Then the agent ends up being indebted at the terminal date. Most agents have to consider the possibility of negative non-market wealth. In this case, the effect of non-market wealth on derived utility is more complex. The effect of a multiplicative background risk on derived relative risk aversion and on portfolio policy is correspondingly complex.

We begin the analysis as before by considering the possible effect of nonmarket wealth on derived relative risk aversion $A_{z}(x)$.

Lemma 3 Suppose $E(\tilde{z})>0$ and $z_{\min }<0$. Then $A_{z}(x) \rightarrow \infty$ as $x \rightarrow-z_{\min }$ and $\exists\left(x^{o}, x^{o o}, x^{o o o}\right)$ with $x^{o} \leq x^{o o} \leq x^{o o o}$ such that:

- $A_{z}(x)$ is declining and convex for $x \leq x^{o}$,

- $A_{z}(x)$ has a minimum at $x^{o o}$, 
- $A_{z}(x)$ is increasing and concave for $x>x^{\text {ooo }}$.

Also, as $x \rightarrow \infty, A_{z}(x) \rightarrow \gamma$.

Proof: See Appendix 2.

The result in Lemma 3 shows that when the expected non-market wealth is positive, but the minimum non-market wealth negative, the behaviour of the derived relative risk aversion, $A_{z}(x)$, is range dependent. First, $A_{z}(x)$ is very high and declines to some level below $\gamma$. Finally, $A_{z}(x)$ approches $\gamma$ as $x$ becomes large. Hence there exists a minimum of $A_{z}(x)$. Hence it could be $\mathrm{U}$-shaped, as illustrated by the numerical example in Figure 1. There is some empirical evidence in support of such a U-shape for relative risk aversion. In particular, Ait-Sahalia and Lo (2000) and Jackwerth (2000) use market data to show that the pricing kernel reflects a relative risk aversion function, of the representative agent which is U-shaped.

In the numerical example in Figure 1, we consider market wealth $x$ in the range $(30,300)$. Non-market wealth is symmetrically distributed around its mean of 30 according to the distribution $(-30,6.25 \% ; 0,6.25 \% ; 30,37.5 \%$; $60,25 \% ; 90,6.25 \%)$. In each pair the first number denotes the realization of $z$ and the second number its probability. Hence $\tilde{z}$ has a standard deviation of 30 and a minimum realization of -30 . For $\gamma=1.5$ the derived relative risk aversion $A_{z}(x)$ limits to infinity for $x=30$ and to 1.5 for large $x$. In between there exists an $x$-range with $A_{z}(x)<\gamma . A_{z}(x)$ is declining and convex for $x<x^{o o}=130$ where it reaches a minimum. After that it increases gradually to 1.5 . For $x>x^{o o o}=196$ it is increasing and concave.

The intuition for this U-shape is as follows. At low levels of market wealth, the addition of a possibly negative non-market wealth dominates. The agent must avoid a negative total wealth at all costs and, hence, risk aversion for the derived utility function will approach infinity as market wealth plus minimal non-market wealth approaches zero. At moderately higher wealth levels, the risk effect of non-market wealth is lower and dominated by the effect of its positive mean. This leads to $A_{z}(x)$ less than 1.5 and increasing, similar to Result 1a in section 3.1. Hence a minimum of $A_{z}(x)$ exists. At very high levels of market wealth, non-market wealth becomes a trivial part 
of total wealth, so that $A_{z}(x)$ asymptotically approaches the level of relative risk aversion without non-market wealth, which is 1.5 in our example.

The extent of the U-shape in the $A_{z}(x)$ function and its significance depend upon the values of $\bar{z}>0$ and $\sigma_{z}$. If $\sigma_{z}$ is small in comparison with the expected value, there may be only a small chance of $z<0$ and consequently only a small $x$-range in which $A_{z}(x)$ declines. If $\sigma_{z}$ is large in comparison with the expected value, the $x$-range in which $A_{z}(x)$ declines may be large.

Lemma 3 and Result 3 imply that the effect of a multiplicative risk on derived relative risk aversion is range dependent. The multiplicative risk raises derived relative risk aversion in the range $x \leq x^{o} / y_{\max }$ and lowers it in the range $x \geq x^{\text {ooo }} / y_{\min }{ }^{13}$ Hence the U-shape of derived relative risk aversion is preserved under the multiplicative risk, as illustrated in Figure 1. The following proposition states the implications for portfolio choice.

Proposition 3 Suppose $E(\tilde{z})>0$ and $z_{\min }<0$. Then non-market wealth induces the agent to behave in a more [less] conservative manner whenever $A_{z}(x)>[<] \gamma$.

The addition of a multiplicative risk induces the agent to behave in a more [less] conservative manner for $x \leq x^{o} / y_{\max }\left[x \geq x^{\text {ooo }} / y_{\min }\right]$, provided that $A_{z}(x y) \geq 1, \forall x y$.

Proposition 3 says first that in this case of positive expected non-market wealth with a negative minimum agents may act in either a more or less conservative manner given non-market wealth, since derived risk aversion $A_{z}(x)$ can be higher or lower than $\gamma$. Also, they may react to a multiplicative background risk by becoming either more or less conservative in their portfolio choice.

There are two interesting implications of Proposition 3. First, in the absence of multiplicative risk, we know that $\sigma_{z}>0$ increases derived relative risk aversion and leads to a more conservative portfolio strategy. On the other

\footnotetext{
${ }^{13}$ This is also illustrated in Figure 1 assuming that $y$ is binomial with realisations 0.7 and 1.3, each with probability 0.5 . Then $x y_{\min }+z_{\min }=0.7 x-30>0$ requires $x>43$ so that $A_{z, y}(x)$ is defined only for $x>43$.
} 
hand, with $\sigma_{z} \rightarrow 0$ and $\bar{z}>0$, Proposition 2 implies that a multiplicative risk, $\sigma_{y}>0$ reduces derived relative risk aversion and makes the agent act in a less conservative manner. Since these two risks have opposite effects, one might expect the combination of the risks to lead to behaviour somewhere between these extremes. However, this need not be the case. The interaction of the two effects is complex. If $\sigma_{z}$ gets large enough so that $z_{\min }$ becomes negative, the derived relative risk aversion, $A_{z}(x)$ changes from being increasing and concave (when $\sigma_{z} \rightarrow 0$ ) to being decreasing and convex, at least over some range of low values of $x$. If $\sigma_{z}$ is large enough, the effect of a multiplicative risk will be to make the agent choose an even more conservative portfolio. Hence, we have an anomaly. The two risks in isolation act in opposite directions, but the two risks together may act in the same direction, re-enforcing each other. This counter-intuitive result illustrates the point that we cannot predict behaviour by considering the non-market wealth risk and the multiplicative risk separately. Whenever non-market wealth risk is large rendering $z_{\text {min }}$ negative, it turns around the way in which the multiplicative risk affects derived relative risk aversion and portfolio policy.

Proposition 3 shows that we should expect complex reactions to the background risks, even if agents have CRRA utility. Observed behaviour can reflect increasing, declining, or U-shaped derived relative risk aversion.

\section{Optimal Contingent Claim Choice: Numer- ical Examples}

In the following, we illustrate the previous results by some numerical examples. Our simulations are based around twelve different scenarios, detailed in Table 1. As we have seen above, the derived risk aversion of an agent and the resulting optimal portfolio demand depend upon three factors, the expected level of non-market wealth, the additive background risk associated with it, and the multiplicative background risk. Expected non-market wealth can be zero (the base case), positive (the most likely case) or negative. The level of the additive background risk, measured by the standard deviation of nonmarket wealth, $\sigma_{z}$, can be positive or zero. The level of the multiplicative background risk, measured by the standard deviation, $\sigma_{y}$, can also be posi- 
tive or zero. Since all combinations are possible, there are a total of twelve possible cases.

In order to illustrate the effects of non-market wealth on portfolio choice, we now present numerical examples for each of the cases above. In Table 2 we summarize the data on which the numerical simulations are based. We assume a binomial market return process evolving over 7 years leading to 8 states of nature at the terminal date. The gross market return in every year is either $90 \%$ or $130 \%$ with equal probability so that the expected annual gross return is $110 \%$ with standard deviation $20 \%$. The annual risk-free rate is $5 \%$ yielding an equity premium of $5 \% .{ }^{14}$ The agent buys state-contingent claims at date $0 .{ }^{15}$

When we derive the agent's static demand, $x(R)$, we present the demand for each of the eight outcomes of the gross market return $R$ at year 7 .

The agent has an initial wealth of 100, and has CRRA preferences with a coefficient of relative risk aversion, $\gamma=1.5$. In the various cases shown below, the expected value of the non-market wealth, $\bar{z}$, takes on values of $-20,0$ and 30 .

Let $\widetilde{z}=\bar{z}+\tilde{\varepsilon}$. Then $\tilde{\varepsilon}$ in our simulation has outcomes $(60,6.25 \% ; 30,25 \%$; $0,37.5 \%$; $-30,25 \%$; $-60,6.25 \%$ ) where the second number in each pair is the probability. Hence, with $\tilde{z}$ risk, the lowest realization of non-market wealth is negative, in all cases. The multiplicative background risk $\widetilde{y}$ is either 0.7 or 1.3 with equal probability.

\subsection{Fixed (Non-Stochastic) Non-Market Wealth}

In Figure 2, we illustrate the optimal solution using the log-demand function from equation (1). We assume that $\gamma=1.5$. The optimal demand function is computed by solving the first-order condition for each state subject to the

\footnotetext{
${ }^{14}$ Hence, the elasticity of the pricing kernel, $\frac{d \ln \phi}{d \ln R}$ is equal to the equity premium, 0.05 , divided by the squared market volatility, 0.04 , or 1.25 .

${ }^{15}$ Of course, a truly dynamic model with learning about $\widetilde{z}$ and $\widetilde{y}$ would be more realistic, but also more complex. Our point here is to show how these risks can affect observed portfolio-choice behavior, even in this simple setting.
} 
budget constraint. We take the three cases from Table 1, where non-market wealth is non-stochastic and multiplicative risk is absent, i.e. cases 1,5, and 9. In case 1 , the expected non-market wealth is $\bar{z}=0$. With $\bar{z}=0$, the relative risk aversion $A(x)=\gamma=1.5$ is a constant. Since the elasticity of the

pricing kernel $\left(-\frac{d \ln \phi}{d \ln R}\right)$ is also a constant, the optimal log-demand for state contingent claims is linear. This is an example of the case in Merton (1971).

Case 9 shows the effect of a positive expected non-market wealth. Here we assume that $\bar{z}=30$. The resulting optimal demand function is steeper (see Figure 2), reflecting the lower derived relative risk aversion. It is also concave reflecting the fact that, in this case, derived relative risk aversion is increasing. Case 5 shows the effect of a negative expected non-market wealth, where $\bar{z}=-20$. In this case, the demand curve is less steep and convex in Figure 2, reflecting greater risk aversion as well as decreasing relative risk aversion for the derived utility.

Using these cases as a starting point, we next examine the effect of either adding risk to non-market wealth, or adding a multiplicative background risk, or both.

\subsection{Background Risks}

In order to delineate the cases, we again consider three alternative scenarios in the presence of background risks: namely the cases where the expected non-market wealth is zero, negative or positive.

\subsubsection{Zero expected non-market wealth}

First consider the case where the non-market wealth is always zero, $\widetilde{z} \equiv 0$. As mentioned previously, adding the background risk $\widetilde{y}$ in this case has no effect on portfolio choice. This is seen in Figure 3 by noting that the base case (with $\widetilde{z} \equiv 0$ ) in case 1 yields identical results to case 2 .

Now consider risky non-market wealth with zero expectation, $\bar{z}=0$. This allows us to focus on pure risk effects. As is known in this case, the agent behaves in a more risk-averse manner when the non-market wealth is risky. 
This is seen in Figure 3 by comparing the base case (with $\widetilde{z} \equiv 0$ ) in case 1 with the case of a positive $\widetilde{z}$ risk in case 3 . In this case, the demand for state contingent claims is flatter, due to the more-risk-averse portfolio choice. Moreover, the demand curve is no longer linear, but rather convex, due to the decreasing relative risk aversion in this case. This is as predicted by result 2 in section 3 .

If we now consider both the $\widetilde{z}$ risk and the $\widetilde{y}$ risk as existing concurrently, it follows from Appendix 2 that behavior will be even more risk averse than under the $\widetilde{z}$ risk alone. Although the $\widetilde{y}$ risk in isolation does not affect behavior, it makes the agent worse off and here we see how it causes the agent to behave as if she were poorer and hence more sensitive to the additional $\widetilde{z}$ risk. Moreover, it follows in this case that $v(x) \equiv E u(x \widetilde{y}+\widetilde{z})$ exhibits decreasing relative risk aversion. ${ }^{16}$ This is illustrated in case 4 of Figure 3 and illustrates Proposition 1.

\subsubsection{Negative expected non-market wealth}

When $\bar{z}<0$ and $\sigma_{z}=0$, behavior appears to be more risk averse than CRRA would indicate as well as exhibiting decreasing relative risk aversion. Adding the background risk $\widetilde{y}$ makes behavior seem even more risk averse. This shows up only slightly in Figure 4 in cases 5 and 6 . This illustrates Results $1 \mathrm{~b}$ and 3 above.

It also follows in Case 7 that adding the $\widetilde{z}$ risk, but in the absence of the $\widetilde{y}$ risk, makes behavior seem more risk averse and also seem to exhibit decreasing relative aversion, as is seen by comparing cases 5 and 7 in Figure 4 . This illustrates Result 2 above.

If we include both the $\widetilde{z}$ risk and the $\widetilde{y}$ risk simultaneously, we see that the effects of more risk averse behavior and of decreasing relative risk aversion are magnified. Case 8 in Figure 4 illustrates this situation.

\footnotetext{
${ }^{16}$ This follows by noting that $\widehat{v}(x) \equiv E u(x+\widetilde{z})$ satisfies decreasing relative risk aversion, as shown in the appendix of this paper. Hence, from Franke, et al (2006, appendix), it follows that adding the $\widetilde{y}$ risk to obtain $v(x) \equiv E u(x \widetilde{y}+\widetilde{z})$ preserves this property.
} 


\subsubsection{Positive expected non-market wealth}

This is perhaps the most realistic and most interesting case. When $\bar{z}>0$, but background risks are absent, the agent appears to behave in a less riskaverse manner than CRRA would indicate. In addition, behavior appears to exhibit increasing relative risk aversion. This is the base case 9 in Figure 5, where the log-demand curve for contingent clams is slightly concave. If we add the multiplicative background risk $\widetilde{y}$, behaviour here becomes slightly less risk averse, as indicated by the slightly steeper demand curve. This is illustrated in case 10 and is as predicted by Result $3 \mathrm{~b} .^{17}$

If a risk $\widetilde{z}$ is added to the non-market wealth in the absence of multiplicative $\widetilde{y}$ risk, the addition of $\widetilde{z}$ increases the level of risk aversion (case 11). Since non-market wealth is negative with positive probability, Lemma 1 applies. Hence, for low levels of market wealth relative risk aversion is higher than $\gamma$, then it declines to a level below $\gamma$ and gradually approaches $\gamma$ for high levels of market wealth. Hard to see in Figure 5 for case 11, for low values of $\ln R$ the log-demand curve for contingent claims is convex, indicating decreasing relative risk aversion. But for high levels of $\ln R$, the demand curve is slightly concave, indicating that relative risk aversion is increasing.

In case 12 , we show the effects of including both the $\widetilde{z}$ risk and the $\widetilde{y}$ risk simultaneously. Although the effect of the $\widetilde{y}$ risk in isolation is to cause a decrease in observed risk aversion, the $\widetilde{y}$ risk also makes the agent more sensitive to the $\tilde{z}$ risk. From Proposition 3 we know that for low levels of market wealth both risks reinforce each other making behavior appear more risk averse than in the presence of the $\widetilde{z}$ risk only. This is evident in Figure 5 from the small slope of the case- 12 curve in the range of low market returns. For high levels of market wealth, the $\widetilde{y}$ risk makes behavior appear less risk averse than in the presence of the $\widetilde{z}$ risk only. Hence in Figure 5 the slope of the case-12 curve is smaller than that of the case- 10 curve in the range of high market returns.

\footnotetext{
${ }^{17}$ The property of increasing relative risk aversion need not always hold in this setting, but it does in this example.
} 


\section{Conclusions}

Risk taking is complicated by the personal circumstances of the agent. Here, we have analyzed the optimal risk taking of a CRRA agent. The agent has stochastic non-market wealth and is also subject to a multiplicative background risk. If we only observe the portfolio choice of the agent, it might be difficult to observe anything that looks similar to her underlying CRRA preferences. The existence of non-market wealth may cause the agent to act as if her utility had increasing or declining relative risk aversion, depending on the size and risk of the non-market wealth. The response to a multiplicative background risk crucially depends upon the mean of non-market wealth and its riskiness .

Consideration of the additive non-market wealth risk and the multiplicative background risk together in one model is important, since the combined effect can be quite different from the effect of one of these risks alone. Consider the case in which non-market wealth has positive expectation but is not risky. Then the effect of the multiplicative risk alone is to increase risk taking, whereas we may see the opposite effect when non-market wealth risk is also risky. Ignoring the interaction effects between the risks can lead to incorrect predictions of the effect of background risks on portfolio policies.

In our model, resolution of the uncertainty surrounding the non-market wealth risk and multiplicative background risks only takes place at the terminal date. We solve a single-period model for the optimal demand function for state-contingent claims. However, if the market for the risky asset is dynamically complete, this function can be represented by a dynamic asset-allocation strategy. This strategy is complicated by background risks, suggesting that simple dynamic strategies are likely to be sub-optimal. 
Table 1: Twelve Cases of Expected Non-Market Wealth, Additive Background Risk and Multiplicative Background Risk

\begin{tabular}{|c|c|c|c|c|}
\hline & $\begin{array}{c}\text { Non-stochastic } \\
\text { non-market wealth } \\
\text { No multiplicative } \\
\text { risk }\end{array}$ & $\begin{array}{c}\text { Non-stochastic } \\
\text { non-market wealth } \\
\text { Multiplicative } \\
\text { risk }\end{array}$ & $\begin{array}{c}\text { Stochastic } \\
\text { non-market wealth } \\
\text { No multiplicative } \\
\text { risk }\end{array}$ & $\begin{array}{c}\text { Stochastic } \\
\text { non-market wealth } \\
\text { Multiplicative } \\
\text { risk }\end{array}$ \\
\hline $\begin{array}{c}\text { Zero-mean } \\
\text { non-market } \\
\text { wealth }\end{array}$ & $\begin{array}{c}\text { Case } 1: \bar{z}=0, \\
\sigma_{y}=0, \sigma_{z}=0\end{array}$ & $\begin{array}{c}\text { Case } 2: \bar{z}=0 \\
\sigma_{y}>0, \sigma_{z}=0\end{array}$ & $\begin{array}{c}\text { Case } 3: \bar{z}=0 \\
\sigma_{y}=0, \sigma_{z}>0\end{array}$ & $\begin{array}{c}\text { Case } 4: \bar{z}=0 \\
\sigma_{y}>0, \sigma_{z}>0\end{array}$ \\
\hline $\begin{array}{c}\text { Negative-mean } \\
\text { non-market } \\
\text { wealth }\end{array}$ & Case 5: $\bar{z}<0$ & Case $6: \bar{z}<0$ & Case $7: \bar{z}<0$ & Case 8: $\bar{z}<0$ \\
$\sigma_{y}=0, \sigma_{z}=0$ & $\sigma_{y}>0, \sigma_{z}=0$ & $\sigma_{y}=0, \sigma_{z}>0$ & $\sigma_{y}>0, \sigma_{z}>0$ \\
\hline $\begin{array}{c}\text { Positive-mean } \\
\text { non-market } \\
\text { wealth }\end{array}$ & Case 9: $\bar{z}>0$ & Case $10: \bar{z}>0$ & Case $11: \bar{z}>0$ & Case $12: \bar{z}>0$ \\
$\sigma_{y}=0, \sigma_{z}=0$ & $\sigma_{y}>0, \sigma_{z}=0$ & $\sigma_{y}=0, \sigma_{z}>0$ & $\sigma_{y}>0, \sigma_{z}>0$ \\
& \multicolumn{2}{|r}{} & & \\
\hline
\end{tabular}

1. $\bar{z}$ is the mean of non-market wealth

2. $\sigma_{z}$ is the standard deviation of the non-market wealth

3. $\sigma_{y}$ is the standard deviation of the multiplicative risk factor 


\section{Table 2: Portfolio Optimisation Example: Data}

\begin{tabular}{|c|c|c|c|c|}
\hline $\begin{array}{l}\text { Annual Expected Gross } \\
\text { Return on Market } \\
\text { Annual Risk-free Rate } \\
\text { Annual Volatility of } \\
\text { Market Return, }\end{array}$ & $\begin{array}{l}1.1 \\
5 \%\end{array}$ & $\begin{array}{l}\text { Coefficient of } \\
\text { Relative Risk aversion, } \\
\text { Expected Non-market } \\
\text { Wealth, } \\
\text { Investible wealth, } \\
\text { Standard deviation of } \\
\text { Non-market wealth } \\
\text { Standard deviation of } \\
\text { Multiplicative risk }\end{array}$ & $\begin{array}{l}\bar{z} \\
x_{0}\end{array}$ & $\begin{array}{l}1.5 \\
-20,0,30 \\
100 \\
0,30 \\
0,0.3\end{array}$ \\
\hline
\end{tabular}

1. We assume that the market return is represented by a discrete binomial process, with a mean return of $10 \%$ over each of the 7 years. The volatility of the underlying process, $\sigma_{m}$, is $20 \%$.

2. The annual risk-free rate of interest is $5 \%$.

3. In the right hand columns we show the agent characteristics. The coefficient of relative risk aversion is $\gamma=1.5$. 
Notes for Figures

In Figures 2-5, the trio $\left(\bar{z}, \sigma_{z}, \sigma_{y}\right)$ signifies the levels of expected non-market wealth, the risk of non-market wealth and the $\widetilde{y}$ risk.

1. Figure 1 depicts the derived RRA for $\gamma=1.5$ and positive fixed non-market wealth $\bar{z}=30$ and the derived RRA for risky non-market wealth with expectation 30. The probability distribution of $z$ is : $z=30 \pm 60$ with probability $1 / 16, z=30 \pm 30$ with probability $4 / 16$, and $z=30$ with probability $6 / 16$. Also, the derived relative risk aversion is shown where $y$ is 0.7 or 1.3 with equal probability. The graph shows the derived relative risk aversion a) in the absence of both additive and multiplicative background risks, b) in the absence of one of the background risks, c) in the presence of both additive and multiplicative background risks.

2. In Figure 2, we plot the logarithm of the portfolio gross return against the logarithm of the market gross return, for the case where there is no nonmarket wealth risk and no $\widetilde{y}$ risk. The expected return on the market is $10 \%$ and the volatility of the market is $20 \%$. The coefficient of relative risk aversion is $\gamma=1.5$. The expected non-market wealth is $\bar{z}=0$ in case 1 , $\bar{z}=-20$ in case 5 , and $\bar{z}=30$ in case 9 .

3. In Figure 3, we compare four different cases. In each case, the expected value of the non-market wealth is $\bar{z}=0$, while the market data and the coefficient of relative risk aversion are the same as in the example in Figure 2. In case 1 we assume that there is no risk, i.e. neither non-market wealth risk nor $\widetilde{y}$ risk. In case 2 , the risk of the non-market wealth is $\sigma_{z}=0$ and the $\widetilde{y}$ risk $\sigma_{y}=0.3$. In case 3 , the risk of the non-market wealth is $\sigma_{z}=30$ and the $\widetilde{y}$ risk $\sigma_{y}=0$. In case 4 , the risk of the non-market wealth is $\sigma_{z}=30$ and the $\widetilde{y}$ risk is $\sigma_{y}=0.3$.

4. In Figure 4, we compare four different scenarios. In each case, the expected value of the non-market wealth is $\bar{z}=-20$, while the market data and the coefficient of relative risk aversion are the same as in the example in Figure 2. In case 5 we assume that there is no risk, i.e. neither non-market wealth risk nor $\widetilde{y}$ risk. In case 6 , the risk of the non-market wealth is $\sigma_{z}=0$ and the $\widetilde{y}$ risk $\sigma_{y}=0.3$. In case 7 , the risk of the non-market wealth is $\sigma_{z}=30$ and 
the $\widetilde{y}$ risk $\sigma_{y}=0$. In case 8 , the risk of the non-market wealth is $\sigma_{z}=30$ and the $\widetilde{y}$ risk is $\sigma_{y}=0.3$.

5. In Figure 5, we compare four different scenarios. In each case, the expected value of the non-market wealth is $\bar{z}=30$, while the market data and the coefficient of relative risk aversion is the same as in the example in Figure 2. In case 9 we assume that there is no risk, i.e. neither non-market wealth risk nor $\widetilde{y}$ risk. In case 10 , the risk of the non-market wealth is $\sigma_{z}=0$ and the $\widetilde{y}$ risk $\sigma_{y}=0.3$. In case 11 , the risk of the non-market wealth is $\sigma_{z}=30$ and the $\widetilde{y}$ risk $\sigma_{y}=0$. In case 12 , the risk of the non-market wealth is $\sigma_{z}=30$ and the $\widetilde{y}$ risk is $\sigma_{y}=0.3$. 


\section{Appendix 1: A Generalized Model to Capture the Dependence of Background Risks on the Market Return}

The above model does not account for possible correlation between the market return and the two background risks. There are good reasons to believe that the multiplicative background rate could be correlated with the market return. For example, in the case where the multiplicative background rate is an annuity rate, it is likely that this rate is correlated with the return on the market portfolio. Also, non-market wealth could depend on similar factors to those that determine market wealth. However, to the extent that these background risks are dependent on the market return, simple adjustments to the market wealth demand, $x(\tilde{R})$, can be made to partially hedge these risks. In this section, we show how the solution to the simple model above can be applied to account for correlation.

To generalize the model, we assume that the consumable wealth is

$$
\tilde{w}=\tilde{X} \tilde{Y}+\tilde{Z}
$$

where $\tilde{X}=X(\tilde{R})$ is the market wealth, $\tilde{Y}=Y(\tilde{R}) \tilde{y}, E \tilde{y}=1$ is the multiplicative risk factor and $\tilde{Z}=Z(\tilde{R}) Y(\tilde{R}) \tilde{y}+\tilde{z}$ is the non-market wealth. In this model, $Y(\tilde{R})$ captures the dependence of the multiplicative risk factor on the market return and $Z(\tilde{R})$ the dependence of non-market wealth on the market return. Here we assume that $\tilde{y}$ and $\tilde{z}$ are independent random variables

In this generalized model, the terminal wealth can therefore be written

$$
\tilde{w}=[X(\widetilde{R})+Z(R)] Y(\tilde{R}) \tilde{y}+\tilde{z},
$$

where $\tilde{z}, \tilde{y}$ and $\tilde{R}$ are independent risks. The agent chooses $X(R)$ to maximize expected utility of wealth subject to a budget constraint

$$
\max _{X(R)} E[u([X(\widetilde{R})+Z(\tilde{R})] Y(\tilde{R}) \tilde{y}+\tilde{z})] \text {, s.t. } E[\hat{\phi}(\widetilde{R}) X(\widetilde{R})]=X_{0},
$$

where $X_{0}$ is the agent's initial endowment of market wealth and $\hat{\phi}(\widetilde{R})$ is the pricing kernel. 
To solve for the optimal demand, $X^{*}(R)$, we substitute $X(R)$ by $x(R)$ defined as

$$
x(R) \equiv[X(R)+Z(R)] Y(R), \forall R,
$$

and then obtain the transformed problem

$$
\max _{x(R)} E[u(\tilde{w})] \text {, s.t. } E\left[\hat{\phi}(\widetilde{R}) \frac{x(\widetilde{R})}{Y(\widetilde{R})}\right]=X_{0}+E[\hat{\phi}(\widetilde{R}) Z(\tilde{R})]
$$

where

$$
\tilde{w}=x(\tilde{R}) \tilde{y}+\tilde{z}
$$

Defining a transformed pricing kernel:

$$
\phi(R) \equiv \hat{\phi}(R) /(Y(R)) / E(1 / Y(\tilde{R})),
$$

and

$$
x_{0} \equiv\left[X_{0}+E(\hat{\phi}(\widetilde{R}) Z(\widetilde{R})] / E(1 / Y(\widetilde{R})),\right.
$$

the transformed problem can be written:

$$
\max _{x(R)} E[u(x(\widetilde{R}) \tilde{y}+\tilde{z})] \text {, s.t. } E[\phi(\widetilde{R}) x(\widetilde{R})]=x_{0}
$$

The transformation of problem (10) into problem (3), which involves $x(\tilde{R}), \tilde{y}$, and $\tilde{z}$, allows us to solve a simpler problem involving background risks which are independent of $\tilde{R}$. The optimal solution $x^{*}(R)$ can then be translated into $X^{*}(R)$ using equation (11). To solve (3), we again define $\nu(x)$ as in (2). In this paper, we therefore concentrate on solving problem (3), for the case where $\tilde{R}, \tilde{z}$ and $\tilde{y}$ are independent risks. 


\section{Appendix 2: Proof of Lemmas}

\section{Proof of Lemma 1:}

Let $A(x+z) \equiv-u^{\prime \prime}(x+z) x / u^{\prime}(x+z)$. Then

$$
\begin{aligned}
A_{z}(x) & =\frac{E\left[-u^{\prime \prime}(x+\tilde{z})\right]}{E\left[u^{\prime}(x+\tilde{z})\right]} x=E\left[\frac{u^{\prime}(x+\tilde{z})}{E u^{\prime}(x+\tilde{z})} A(x+\tilde{z})\right] \\
& \equiv E^{Q}[A(x+\tilde{z})]=\gamma E^{Q}\left(\frac{x}{x+\tilde{z}}\right) .
\end{aligned}
$$

$A_{z}(x) \rightarrow \infty$ for $x \rightarrow-z_{\text {min }}$, since $A\left(x+z_{\text {min }}\right)$ goes to infinity. If $x \rightarrow$ $\infty, A_{z}(x) \rightarrow \gamma$.

Claim 1: Given the conditions of Lemma $1, A_{z}(x)$ is declining.

\section{Proof of claim 1}

Let $\bar{z} \equiv E(\tilde{z})$ and define $\widehat{v}(x) \equiv u(x+\bar{z})$. Then $\widehat{v}(x)$ exhibits standard risk aversion and either constant or decreasing relative risk aversion. Define $v(x)=E u(x+\tilde{z})$. Then

$$
v^{\prime}(x)=E \widehat{v}^{\prime}(x+\tilde{z}-\bar{z}) \equiv \widehat{v}^{\prime}(x-\psi),
$$

where $\psi$ denotes Kimball's (1990) precautionary premium for $\tilde{z}-\bar{z}$.

Relative risk aversion for $v(x)$ is then calculated as

$$
A_{z}(x)=-\frac{x \widehat{v}^{\prime \prime}(x-\psi)\left(1-\psi^{\prime}\right)}{\widehat{v}^{\prime}(x-\psi)}=\widehat{A}(x-\psi) \cdot \frac{\left(1-\psi^{\prime}\right)}{1-\frac{\psi}{x}}
$$

where

$$
\widehat{A}(x-\psi) \equiv-\frac{\widehat{v}^{\prime \prime}(x-\psi)}{\widehat{v}^{\prime}(x-\psi)}(x-\psi)
$$

Since $\widehat{v}$ exhibits standard risk aversion, we know from Kimball (1993) that $\psi^{\prime}(x)$ is negative. Moreover, from (16) we see that $\psi<x$, so that $1-\frac{\psi}{x}>0$.

Differentiating (17)

$$
A_{z}^{\prime}(x)=\widehat{A}^{\prime}(x-\psi) \cdot \frac{\left(1-\psi^{\prime}\right)^{2}}{1-\frac{\psi}{x}}
$$




$$
+\widehat{A}(x-\psi) \cdot \frac{\left(-\psi^{\prime \prime}\right)\left(1-\frac{\psi}{x}\right)+\left(1-\psi^{\prime}\right)\left(\frac{x \psi^{\prime}-\psi}{x^{2}}\right)}{\left(1-\frac{\psi}{x}\right)^{2}} .
$$

The first term on the right-hand side in (18) is non-positive by the assumptions. The second term is negative since $\psi^{\prime \prime}>0$, which follows from Franke et al (1998, Lemma 2). Hence, $A_{z}^{\prime}(x)<0$.

Claim 2: Given the conditions of Lemma 1, $A_{z}(x)$ is convex.

\section{Proof of claim 2}

First, we show that if $u$ is a HARA-utility function with $\gamma>0$, then $\varphi^{\prime}(x)<$ $0, \varphi^{\prime \prime}(x)>0$ and $\varphi^{\prime \prime \prime}(x)<0$.

Franke et al (1998) have shown $\varphi^{\prime}(x)<0$ and $\varphi^{\prime \prime}(x)>0$. Therefore $\varphi^{\prime \prime \prime}(x)<$ 0 remains to be shown. For notational convenience, let $\nu=x+\bar{z}$ and $\tilde{z}-\bar{z}=\sigma \tilde{\eta}$, where $\tilde{\eta}$ is a random variable with mean zero and unit variance. We have

$$
(\nu-\psi)^{-\gamma}=E\left[(\nu+\tilde{z}-\bar{z})^{-\gamma}\right]
$$

or

$$
\left(1-\frac{\psi}{\nu}\right)^{-\gamma}=E\left[\left(1+\frac{\sigma \tilde{\eta}}{\nu}\right)^{-\gamma}\right] .
$$

For a given $\eta$-distribution, it follows that

$$
\frac{\psi}{\nu}=f\left(\frac{\sigma}{\nu}\right)
$$

or

$$
\psi=\nu f\left(\frac{\sigma}{\nu}\right)
$$

Differentiating w.r.t. $\nu$

$$
\begin{aligned}
\psi_{\nu} & =f\left(\frac{\sigma}{\nu}\right)+\nu f^{\prime}\left(\frac{\sigma}{\nu}\right) \frac{-\sigma}{\nu^{2}} \\
& =f\left(\frac{\sigma}{\nu}\right)-f^{\prime}\left(\frac{\sigma}{\nu}\right) \frac{\sigma}{\nu}
\end{aligned}
$$

and differentiating w.r.t. $\sigma$

$$
\psi_{\sigma}=\nu f^{\prime}\left(\frac{\sigma}{\nu}\right) \frac{1}{\nu}=f^{\prime}\left(\frac{\sigma}{\nu}\right)
$$


Hence

$$
\psi_{\nu}=f\left(\frac{\sigma}{\nu}\right)-\psi_{\sigma} \frac{\sigma}{\nu}
$$

Differentiating again w.r.t. $\nu$

$$
\psi_{\nu \nu}=\frac{\partial \psi_{\nu}}{\partial \frac{\sigma}{\nu}} \frac{\partial \frac{\sigma}{\nu}}{\partial \nu}=\frac{\partial \psi_{\nu}}{\partial \frac{\sigma}{\nu}}\left(-\frac{\sigma}{\nu^{2}}\right)
$$

and differentiating again w.r.t. $\sigma$

$$
\psi_{\nu \sigma}=\frac{\partial \psi_{\nu}}{\partial \frac{\sigma}{\nu}} \frac{\partial \frac{\sigma}{\nu}}{\partial \sigma}=\frac{\partial \psi_{\nu}}{\partial \frac{\sigma}{\nu}}\left(\frac{1}{\nu}\right)
$$

It follows that

$$
\psi_{\nu \nu}=\psi_{\nu \sigma}\left(-\frac{\sigma}{\nu}\right)
$$

which is a function of $\sigma / \nu$. Hence,

$$
\psi_{\nu \nu \nu}=\frac{\partial \psi_{\nu \nu}}{\partial \frac{\sigma}{\nu}} \frac{\partial \frac{\sigma}{\nu}}{\partial \nu}=\frac{\partial \psi_{\nu \nu}}{\partial \frac{\sigma}{\nu}} \frac{-\sigma}{\nu^{2}}
$$

and

$$
\psi_{\nu \nu \sigma}=\frac{\partial \psi_{\nu \nu}}{\partial \frac{\sigma}{\nu}} \frac{\partial \frac{\sigma}{\nu}}{\partial \sigma}=\frac{\partial \psi_{\nu \nu}}{\partial \frac{\sigma}{\nu}} \frac{1}{\nu}>0 .
$$

Positivity of $\psi_{\nu \nu \sigma}$ is shown in Franke et al (1998, Lemma 3), and hence

$$
\psi_{\nu \nu \nu}=\psi_{\nu \nu \sigma}\left(-\frac{\sigma}{\nu}\right)<0
$$

This is equivalent to $\psi^{\prime \prime \prime}<0$, q.e.d.

Now we show that $A_{z}^{\prime \prime}(x)>0$.

In the HARA case,

$$
A_{z}(x)=\gamma \frac{1-\psi^{\prime}(x)}{x+\bar{z}-\psi(x)} x
$$

Differentiating w.r.t. $x$ we have

$$
A_{z}^{\prime}(x)=-\gamma \frac{\psi^{\prime \prime}(x) x}{x+\bar{z}-\psi(x)}+\frac{A_{z}(x)}{\gamma x}\left[\gamma-A_{z}(x)\right] .
$$


Differentiating the first term of (19) w.r.t. $x$ yields

$$
-\gamma \frac{\psi^{\prime \prime \prime}(x) x}{x+\bar{z}-\psi(x)}+\frac{\psi^{\prime \prime}(x)}{x+\bar{z}-\psi(x)}\left[A_{z}(x)-\gamma\right],
$$

which is positive, since $\psi^{\prime \prime \prime}(x)<0$, as shown above. Also, the second term in (19) clearly increases with $x$, since $A_{z}^{\prime}(x)<0$. Hence, $A_{z}^{\prime \prime}(x)>0$.

\section{Proof of Lemma 2}

To prove Lemma 2, part 1, first, note from equation (15) $A_{z}(x)<\gamma, \forall x$. Second, we show that $A_{z}(x) \rightarrow 0$ for $x \rightarrow 0$. As

$$
A_{z}(x)=x E^{Q}\left(\frac{\gamma}{x+\tilde{z}}\right)
$$

and $x+\tilde{z}>0, A_{z}(x) \rightarrow 0$ for $x \rightarrow 0$.

Next, we show that $A_{z}(x)$ is increasing and concave for $x \leq x^{o}$. In equation (19), the first term goes to zero for $x \rightarrow 0$, while the second term is positive. The latter follows from

$$
\frac{A_{z}(x)}{\gamma x}=E^{Q}\left(\frac{1}{x+\tilde{z}}\right)>0
$$

and $\left[\gamma-A_{z}(x)\right] \rightarrow \gamma$ for $x \rightarrow 0$. Hence, $A_{z}^{\prime}(x)>0$ for $x \rightarrow 0$.

$A_{z}^{\prime \prime}(x)$ is given by (20) plus the derivative of $E^{Q}(x+\tilde{z})^{-1}\left[\gamma-A_{z}(x)\right]$ with respect to $x$. For $x \rightarrow 0$, the first term in (20) goes to zero while the second term is negative. Also, $E^{Q}(x+\tilde{z})^{-1}\left[\gamma-A_{z}(x)\right]$ declines in $x$ since each factor is positive and declining. Hence, $A_{z}^{\prime \prime}(x)<0$ for $x \leq x^{o}$.

Third, we show that $A_{z}(x)$ is increasing and convex for $x \geq x^{o o}$. Given a CRRA-utility function,

$$
\begin{aligned}
A_{z}(x) & =\gamma \frac{E(x+\tilde{z})^{-\gamma-1}}{E(x+\tilde{z})^{-\gamma}} x \\
& =\gamma \frac{[x+\bar{z}-\varphi]^{-\gamma-1}}{[x+\bar{z}-\varphi]^{-\gamma}}\left(1-\frac{\partial \varphi}{\partial x}\right) x
\end{aligned}
$$


Since we now consider large values of $x$, we may regard $\tilde{\varepsilon}=\tilde{z}-\bar{z}$ as a small risk in the sense of Pratt (1964). Technically, divide $x+\tilde{z}$ by a large positive constant $c$ so that $\sigma(\tilde{\varepsilon} / c)$ is a small risk. Then, dropping $c$ for notational simplicity, for a large $x$ the precautionary premium is given by

$$
\varphi(\tilde{\varepsilon} \mid x) \approx \frac{\gamma+1}{x+\bar{z}} \frac{\sigma^{2}(\tilde{\varepsilon})}{2}
$$

so that

$$
\frac{\partial \varphi}{\partial x} \approx-\frac{\varphi}{x+\bar{z}}
$$

Hence,

$$
\begin{aligned}
A_{z}(x) & =\gamma \frac{1+\varphi /(x+\bar{z})}{x+\bar{z}-\varphi} x \\
& =\gamma \frac{x}{x+\bar{z}} \frac{x+\bar{z}+\varphi}{x+\bar{z}-\varphi} \\
& =\gamma \frac{x}{x+\bar{z}} \frac{1+\frac{\gamma+1}{2} \sigma^{2}(\tilde{\varepsilon})(x+\bar{z})^{-2}}{1-\frac{\gamma+1}{2} \sigma^{2}(\tilde{\varepsilon})(x+\bar{z})^{-2}}
\end{aligned}
$$

For large values of $x$, the second fraction converges much faster to 1 than the first fraction because the second fraction depends on $(x+\bar{z})^{-2}$. Therefore, $A_{z}(x) \rightarrow \gamma \frac{x}{x+\bar{z}}<\gamma$ and finally to $\gamma$. Hence, $A_{z}(x)$ is increasing for large values of $x$.

Finally, in the last equation the first fraction is concave in $x$ while the second is convex. Again, for high values of $x$, the first fraction "dominates" the second, which moves much faster to 1 . Hence, $A_{z}(x)$ is increasing and concave for $x \geq x^{o o}$.

To prove Lemma 2, part 2, note that for a small $\sigma_{z}$ and positive $\bar{z}$, relative risk aversion is similar to that of a HARA function without non-market wealth risk. It follows that the relative risk aversion of the derived utility function is increasing and concave. 


\section{Proof of Lemma 3}

$$
A_{z}(x)=E\left[\frac{u^{\prime}(x+\tilde{z})}{E u^{\prime}(x+\tilde{z})} \frac{x}{x+\tilde{z}}\right] \equiv \gamma E^{Q}\left(\frac{x}{x+\tilde{z}}\right)
$$

Hence, $A_{z}(x) \rightarrow \infty$ for $x \rightarrow \underline{x}=-z_{\min }$ where $z_{\min }$ is the minimal value of $z$ with positive probability (density). Also, $A_{z}^{\prime}(x) \rightarrow-\infty$ for $x \rightarrow \underline{x}$. Also, $A_{z}(x) \rightarrow \gamma$ for $x \rightarrow \infty$. Since $A_{z}(x)>0$ for $x>\underline{x}, A_{z, \varepsilon}^{\prime \prime}(x)>0$ is implied for some range $x \in\left(\underline{x}, x^{\circ}\right)$ with $x^{\circ}>\underline{x}$.

Next, by the same argument as used in Lemma 2, part 1, $A_{z}(x)$ is increasing and concave for large values of $x$. This implies a) that there exists a finite $x^{\circ \circ}$ at which $A_{z}(x)$ attains a minimum, and b) there exists some $x^{000}>x^{0 \circ}$ such that $A_{z}(x)$ is increasing and concave in $x$ for $x>x^{\circ 00}$. 


\section{References}

1. Aït-Sahalia, Y. and A.W. Lo, (2000), "Nonparametric Risk Management and Implied Risk Aversion," Journal of Econometrics 94, 9-51.

2. Back, K. and P. H. Dybvig, (1993), "On Existence of Optimal Portfolios in Complete Markets," Washington University in St. Louis, Working Paper.

3. Bodie, Z., Merton, R. and Samuelson, W.F. (1992), "Labor Supply Flexibility and Portfolio Choice in a Life Cycle Model," Journal of Economic Dynamics and Control, 16, 427-449.

4. Brennan, M.J. and Xia, Y. (2002), "Dynamic Asset Allocation under Inflation," Journal of Finance, 57, 1201-1238.

5. Campbell, J. and Viceira, L. (2001), "Who Should Buy Long-Term Bonds", American Economic Review, 91, 99-127.

6. Eeckhoudt, L., Gollier, C. and Schlesinger, H. (1996), "Changes in Background Risk and Risk Taking Behaviour", Econometrica, 64, 683-689.

7. Franke, G., Schlesinger, H. and Stapleton, R.C. (2006), "Multiplicative Background Risk", Management Science, 52, 146-153.

8. Franke, G., Stapleton, R.C. and Subrahmanyam, M.G. (1998), "Who Buys and Who Sells Options: The Role of Options in an Economy with Background Risk," Journal of Economic Theory, 82, 89-109.

9. Gollier, C. (2001), The Economics of Risk and Time, MIT Press.

10. Gollier, C. and Pratt, J. (1996), "Risk Vulnerability and the Tempering Effect of Background Risk," Econometrica 64, 1109-1124.

11. Jackwerth, J.C., (2000), "Recovering Risk Aversion from Option Prices and Realized Returns," Review of Financial Studies 13, 433-451.

12. Kihlstrom R.E., Romer D. and Williams S. (1981), "Risk Aversion with Random Initial Wealth", Econometrica, 49, 911-920.

13. Kimball, M.S. (1990), "Precautionary Saving in the Small and in the Large", Econometrica, 58, 53-73.

14. Kimball, M.S. (1993), "Standard Risk Aversion", Econometrica, 61, 589-64. 
15. Merton, R. (1971), "Optimal Consumption and Portfolio Rules in a ContinuousTime Model," Journal of Economic Theory 3, 373-413.

16. Nachman, D.C. (1982), "Preservation of 'More Risk Averse' under Expectations", Journal of Economic Theory, 28, 361-368.

17. Pratt, J.W. (1964), "Risk Aversion in the Small and in the Large", Econometrica, 32, 122-136.

18. Ross, S.A. (1981) "Some Stronger Measures of Risk Aversion in the Small and the Large with Applications", Econometrica, 49, 621-638. 


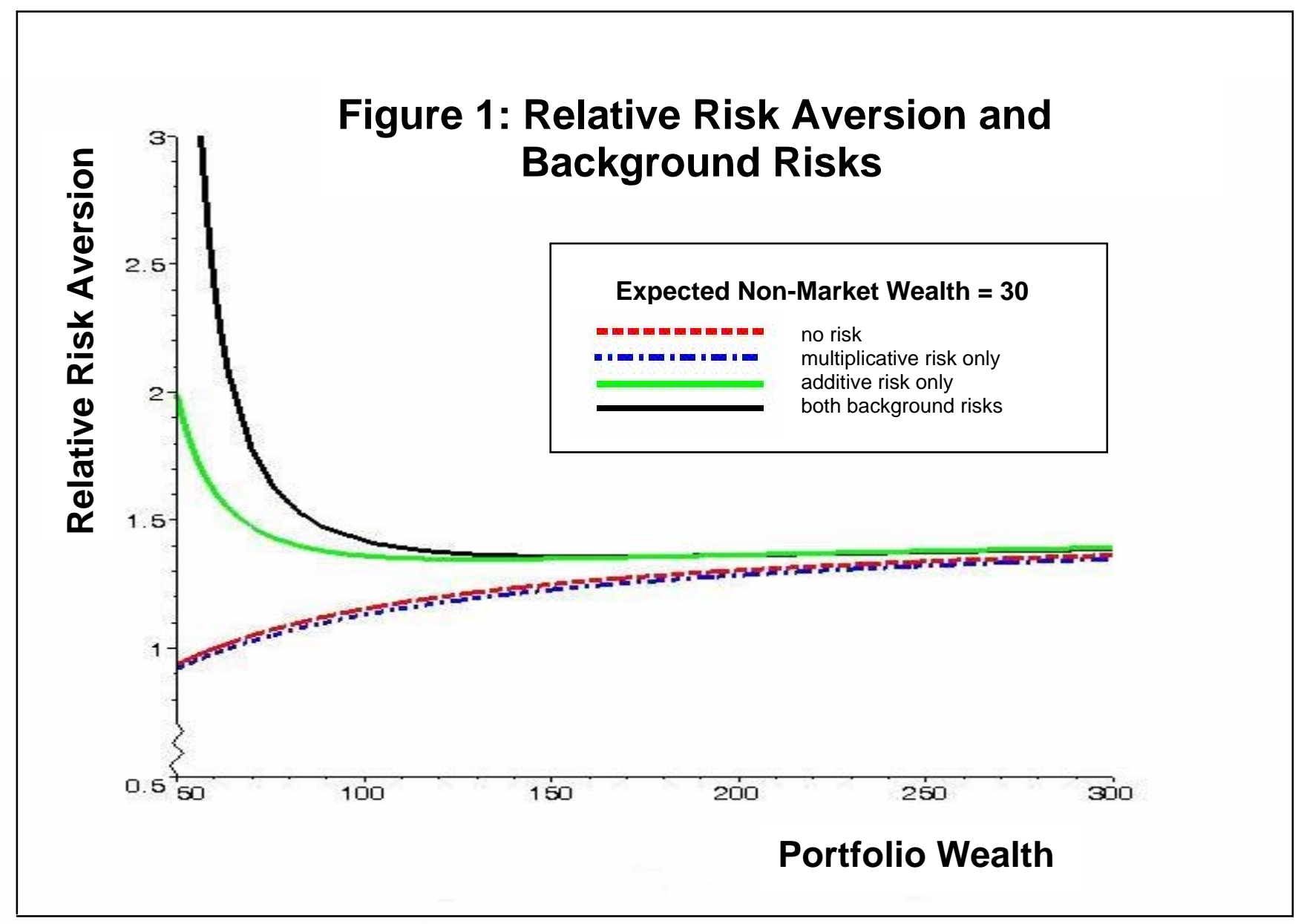




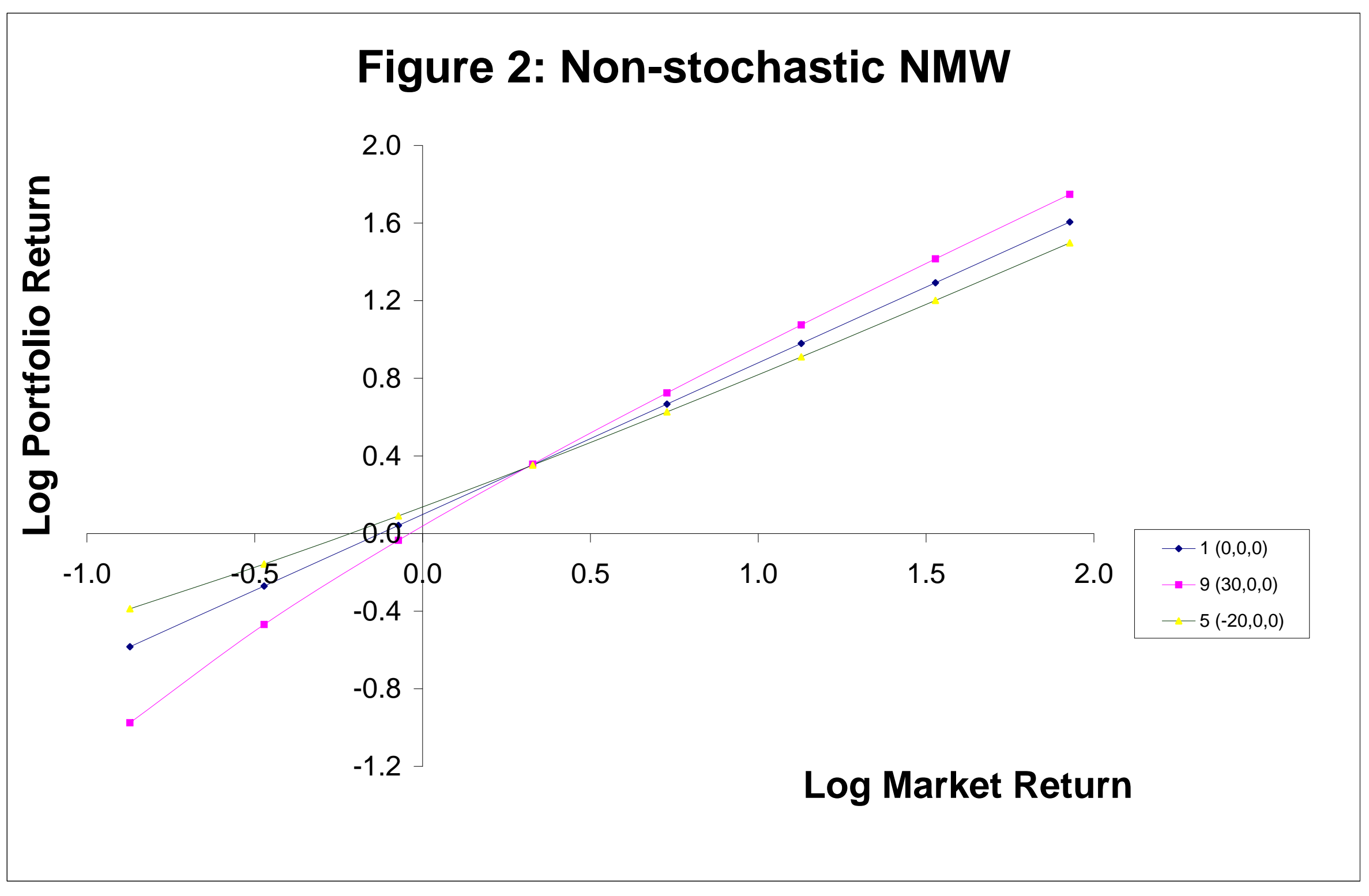




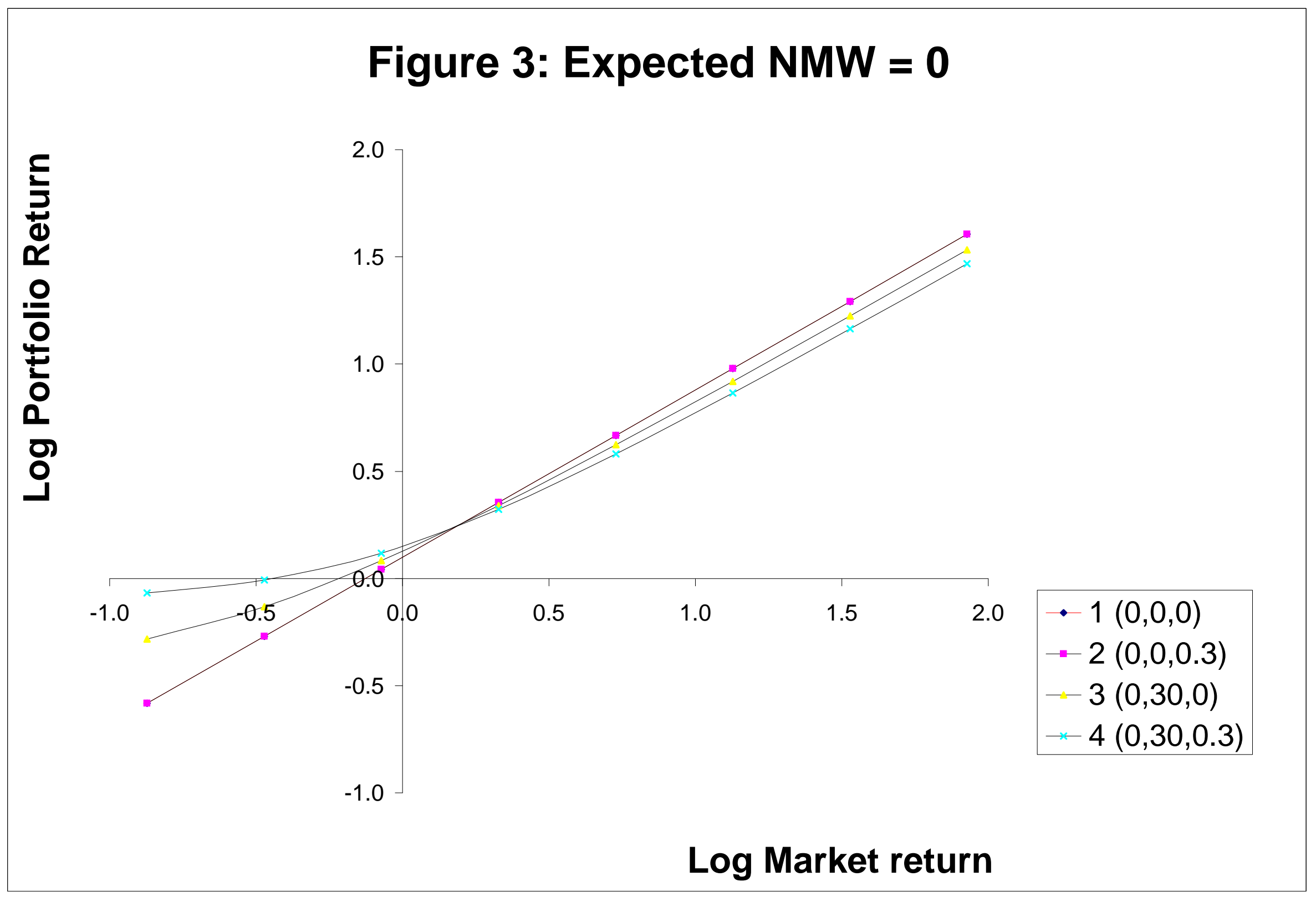




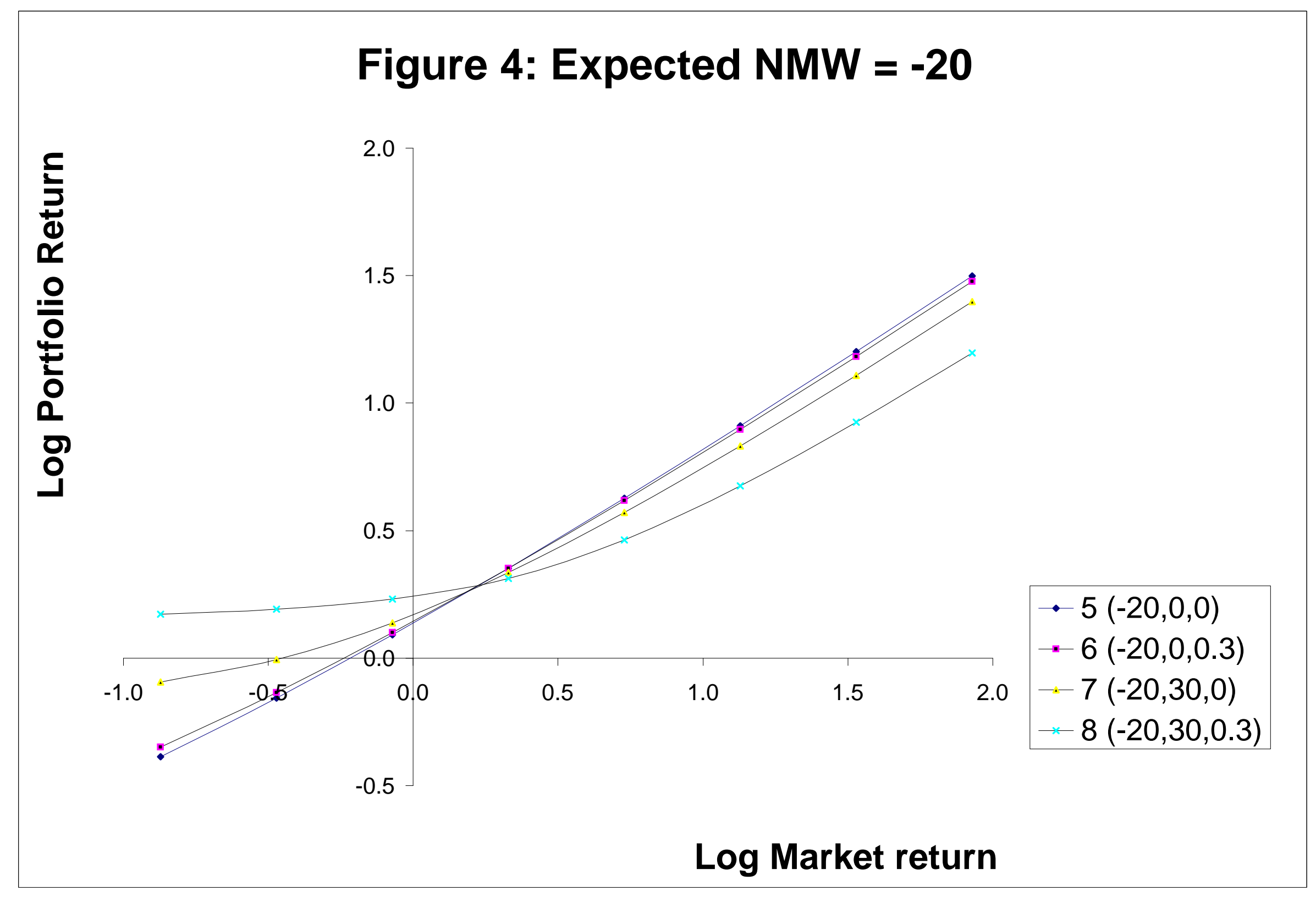




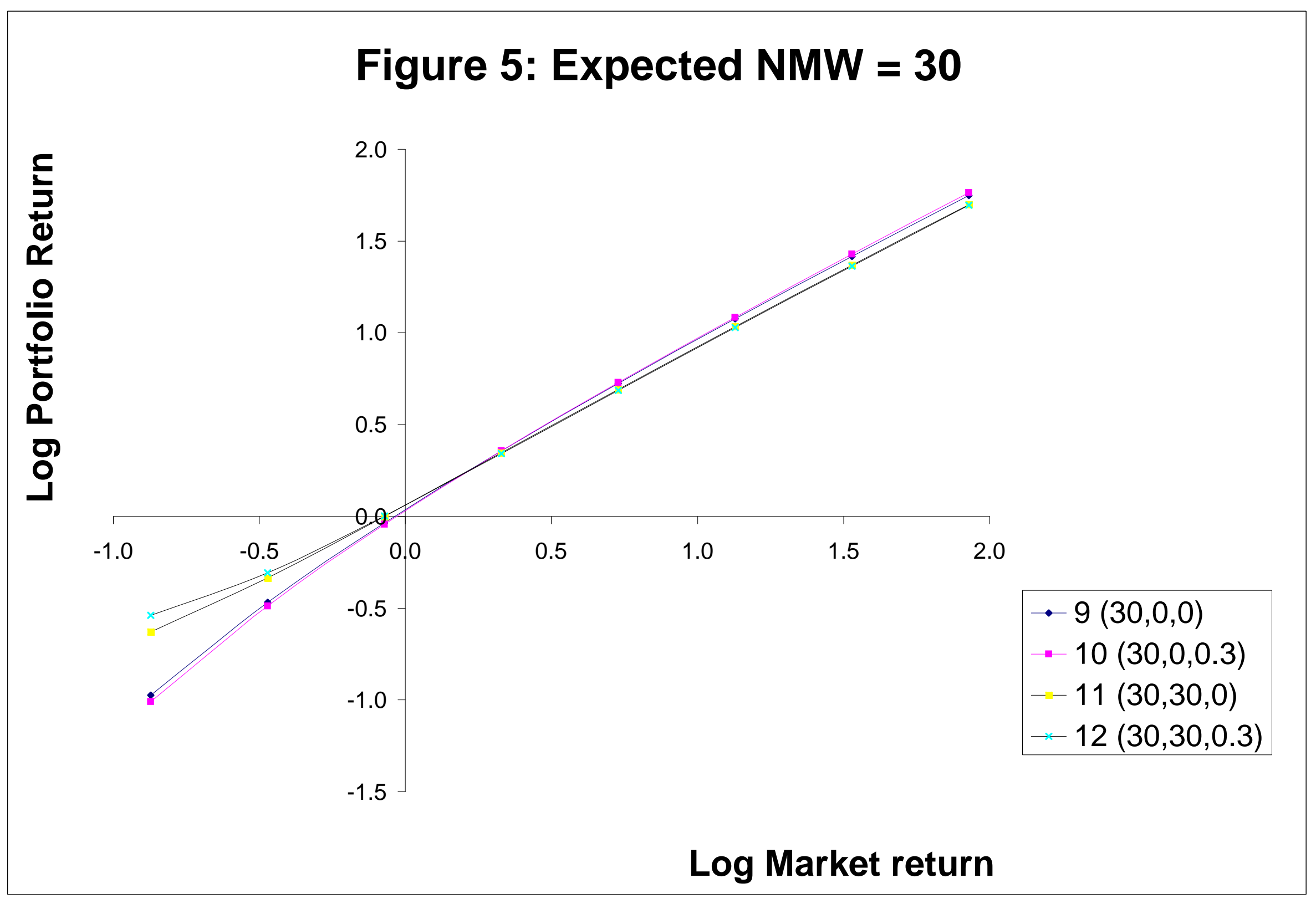

